\title{
205 AGENT-797, A NATIVE ALLOGENEIC 'OFF-THE-SHELF' INKT CELL THERAPY PRODUCT SHOWS ANTI-TUMOR ACTIVITY IN PRECLINICAL XENOGRAFT MODELS
}

${ }^{1}$ Burcu Yigit*, ${ }^{1}$ Darrian Moskowitz, ${ }^{1}$ Xavier Michelet, ${ }^{2}$ Antoine Tanne, ${ }^{1}$ Marc Van Dijk. ${ }^{1}$ Mink Therapeutics, Lexington, MA, USA; ${ }^{2}$ Agenus, Lexington, MA, USA

\subsection{6/jitc-2021-SITC2021.1}

Background agenT-797 is an allogeneic, native invariant natural killer $\mathrm{T}$ (iNKT) cell therapy product currently in phase I clinical trials for cancer (heme and solid). iNKT cells are a distinct population of $\mathrm{T}$ cells that can recognize tumors via direct recognition of CD1d (an MHC-I like molecule presenting glycolipids) through the TCR or recognition of NK cell receptor ligands via various NK receptors. We developed agenT-797 from isolated and ex-vivo expanded peripheral blood iNKT cells. Here we describe in vivo xenograft models to demonstrate the overall tissue distribution, tumor infiltration and efficacy of agenT-797 in liquid as well as solid tumors.

Methods We utilized NOG-hIL15 (human IL-15) transgenic mice to ensure persistence/maintenance of ex-vivo expanded human iNKT cells throughout the studies. For studying efficacy in liquid tumors, we used NALM6, an acute lymphoblastic leukemia (ALL) cell line and for solid tumors selected A375, a melanoma cell line. Both cell lines were engineered to overexpress CD1d. Upon injection of iNKT cells, tumor growth and iNKT cell tissue/tumor infiltration as well as phenotype were studied.

Results Injection of iNKT cells in NALM6- engrafted NOGhIL15 mice resulted in an overall reduction in leukemic burden as measured by luminescence-based imaging. Flow cytometric analysis revealed infiltration of iNKT cells at the site of leukemic expansion, namely blood, spleen, bone marrow and liver. Cells were activated when reaching the site of the tumor. In addition, iNKT cells produced IFN $\gamma$ and TNF $\alpha$ and low levels of IL-13/IL-4, consistent with a Th1 response. When iNKT cells were injected into A375 engrafted mice we observed infiltration of iNKT cells into the tumor, where they become activated and proliferate overtime. We observed an overall reduction in tumor size when iNKT cells were injected compared to control group, demonstrating the impact of iNKT cells on tumor growth.

Conclusions We established xenograft mouse models to address various biological questions around human iNKT cells as a cell therapy product. We have demonstrated homing and infiltration of iNKT cells at the site of tumor and relative proliferation and expansion. These models provide a suitable platform for in-vivo preclinical studies on agenT -797 in cancer.

Ethics Approval All procedures performed in studies involving human participants were in accordance with the ethical standards of the institutional and/or national research committee and with the 1964 Helsinki declaration and its later amendments or comparable ethical standards.

http://dx.doi.org/10.1136/jitc-2021-SITC2021.205 